\title{
Safety of Active Implantable Devices During MRI Examinations: A Finite Element Analysis of an Implantable Pump
}

\author{
Philippe Büchler*, Anne Simon, Jürgen Burger, Alec Ginggen, Rocco Crivelli, Yanik Tardy, \\ Roger Luechinger, and Sigbjørn Olsen
}

\begin{abstract}
The goal of this study was to propose a general numerical analysis methodology to evaluate the magnetic resonance imaging (MRI)-safety of active implants. Numerical models based on the finite element (FE) technique were used to estimate if the normal operation of an active device was altered during MRI imaging. An active implanted pump was chosen to illustrate the method. A set of controlled experiments were proposed and performed to validate the numerical model. The calculated induced voltages in the important electronic components of the device showed dependence with the MRI field strength. For the MRI radiofrequency fields, significant induced voltages of up to $20 \mathrm{~V}$ were calculated for a $0.3 \mathrm{~T}$ field-strength MRI. For the 1.5 and 3.0T MRIs, the calculated voltages were insignificant. On the other hand, induced voltages up to $11 \mathrm{~V}$ were calculated in the critical electronic components for the 3.0T MRI due to the gradient fields. Values obtained in this work reflect to the worst case situation which is virtually impossible to achieve in normal scanning situations. Since the calculated voltages may be removed by appropriate protection circuits, no critical problems affecting the normal operation of the pump were identified. This study showed that the proposed methodology helps the identification of the possible incompatibilities between active implants and MR imaging, and can be used to aid the design of critical electronic systems to ensure MRI-safety.
\end{abstract}

Index Terms-Biomedical equipment safety, finite element methods, magnetic resonance imaging.

\section{INTRODUCTION}

A CCORDING to the U.S. Food and Drug Administration, magnetic resonance imaging (MRI) scanning is contraindicated for patients with active implants such as pacemakers, defibrillators, or neurostimulators [1]-[4]. The main potential hazards that arise include device motion (force,

Manuscript received October 27, 2005; revised September 3, 2006. This work was supported in part by the Swiss innovation promotion agency under Grant CTI \#7053.1MTS-LS. Asterisk indicates corresponding author.

*P. Büchler is with the MEM Research Center, Institute for Surgical Technology \& Biomechanics, University of Bern, CH-3014 Bern, Switzerland (e-mail: philippe.buechler@memcenter.unibe.ch).

A. Simon and J. Burger are with the Institute of Micro-technique and Medical Technology, School of Engineering and Information Technology, CH-2501 Biel, Switzerland.

A. Ginggen, R. Crivelli, and Y. Tardy are with Codman Neurosciences SARL, CH-2560 Le Locle, Switzerland.

R. Luechinger is with the Institute for Biomedical Engineering, University and ETH Zurich, CH-8092 Zürich, Switzerland.

S. Olsen is with the MEM Research Center, Institute for Surgical Technology

\& Biomechanics, University of Bern, CH-3014 Bern, Switzerland.

Digital Object Identifier 10.1109/TBME.2006.890145 torque), device and tissue heating and alteration of the device's operation. Additionally, the imaging artifacts caused by the device may reduce the clinical effectiveness of the MRI procedure.

Assessing whether an implanted device can be safely used in conjunction with MRI is a complex problem. It is relatively easy, for example, to demonstrate that heating or induced voltages can occur, but far more difficult to prove that they are not hazardous. Experimental studies have been extensively used to assess the MRI-safety of various active implants (pacemakers [4]-[9], defibrillators [1], [5], neurostimulators [10], [11], and infusion pumps [2], [12], [13]) and this type of investigation is mandatory before the commercialization and marketing of implants as MRI safe. However, experimental tests are only possible at the end of the product development cycle when fully functional implants are available and design change costs are the highest. Numerical analyses are not constrained to this schedule and are routinely performed, but as of yet, they have only been used to determine the specific absorption rates (SARs) of tissues surrounding the device [14]-[17] and are hence of limited use in evaluating the device's safety. This paper presents a general numerical analysis methodology that enables more comprehensively evaluation of the safety of implantable devices earlier in the product development cycle.

The goal of this study was to develop a methodology for preliminary evaluation of the MRI-safety of active implants. In this paper, we focused on the device operation and, in particular, on whether there was potential for failure of electronic components, because this problem is unique to active implants. The methodology is based on finite element (FE) principles and is illustrated by the evaluation of an active implantable pump. This particular device is interesting in that it includes a telemetric external control system that will likely become more common in the future. The interaction between the antenna of this system and the MRI field may create problems. This work reports on the validation of the FE model with controlled experiments as well as estimation of the MRI-induced voltages for the critical electronic components of the device.

\section{MATERIAL AND MethodS}

\section{A. Implantable Pump}

In this paper, an active implantable pump (Codman Neurosciences SARL, Le Locle, Switzerland) was used as the subject of the numerical analysis of MRI-safety. This pump is used as an implanted drug delivery system for the management of chronic 
pain. It is surgically placed in the abdominal region and delivers drugs directly into the spinal canal. The drug infusion profile can be programmed telemetrically at any time after its implantation using radiofrequency (RF) signals. Data relevant to the therapy, which is stored in the pump, can also be retrieved in this manner. Two electronic modules are of principal consideration in the model and these are the two large coils of the pump. One is the antenna used for communication, and the other is a component of the fill-level sensor, used to monitor the amount of drug in the pump's tank.

\section{B. Assumptions}

During MRI procedures, the electrical components of an implanted device are exposed to two time-varying electromagnetic fields. These include the $\mathrm{B}_{1} \mathrm{RF}$ field that is used to orient the spin axis of polar molecules away from the main MRI axis, and the gradient fields that are used to encode spatial position into the signal for image reconstruction. This work will evaluate the effect that both of these fields have on the pump's electrical components.

The resonance equation, which relates the resonance frequency $\nu$ of a spin to the main magnetic $\mathrm{B}_{0}$, is the following:

$$
\nu=\gamma \mathbf{B}_{0}
$$

where $\gamma$ is the gyromagnetic ratio. This ratio is equal to 42.58 $\mathrm{MHz} / \mathrm{T}$ for hydrogen protons, which are common in biological tissues. For a 1.5T MRI the resonance frequency for hydrogen corresponds to an electromagnetic wavelength of greater than $4.7 \mathrm{~m}$. Since this wavelength is about 60 times the size of the pump being studied $(<80 \mathrm{~mm})$, a low-frequency approximation could be applied to the Maxwell equations used in the calculations. This approximation implies that electromagnetic waves will propagate instantaneously thru the sample. Therefore, the $\mathrm{RF}$ magnetic field is considered to be homogenous over the volume of the MRI device.

\section{FE Simulations of MRI Induced Voltages}

An axisymmetric FE model representing the pump within an MRI electromagnetic environment was developed. This model included the Codman pump that was surrounded by air (see the discussion for the reasoning) and filled with fluid in the lower compartment to simulate the drug. The antenna of the pump was embedded in polyethylene on the outside of the pump casing (Fig. 1). 13000 8-node electromagnetic elements were used in the model. This large number of elements was required to model the eddy currents that occur in only a small fraction of the thickness of metallic components. For example, in the titanium case with a $64 \mathrm{MHz}$ excitation signal, the depth of the eddy current effect is $0.5 \mathrm{~mm}$. In order to obtain accurate results for the metallic components, a higher mesh density was used on the outer surfaces.

A simplified circuit was also coupled to the FE model to model the basic behavior of the electronic circuit (Fig. 1). The antenna and fill-level sensor were both connected to an idealized capacitor element. The capacitance was set for an antenna

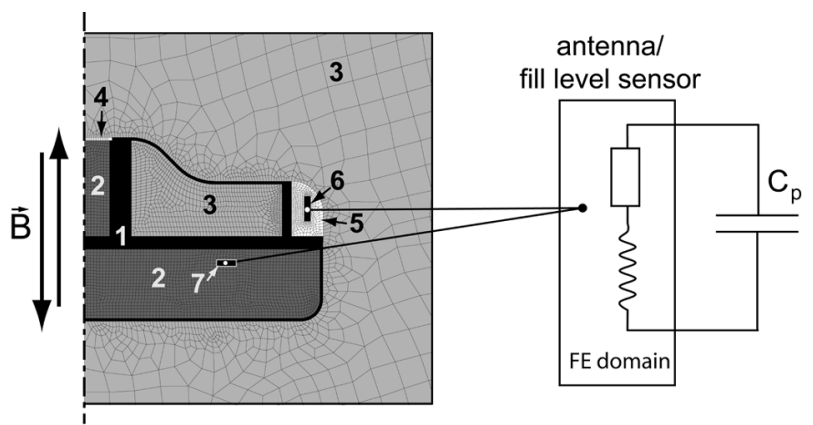

Fig. 1. Detail of the axisymmetric FE mesh used for the simulations. Boundary conditions were chosen to model an oscillating magnetic field in the vertical direction. The numbers correspond to different materials: 1 -titanium casing $\left(\mu_{\mathrm{r}}=1.00018, \rho=5610^{-8} \Omega \cdot \mathrm{m}\right), 2-\operatorname{drug} \operatorname{tank}\left(\mu_{\mathrm{r}}=1, \rho=1.6 \Omega \cdot \mathrm{m}\right)$, 3-air $\left(\mu_{\mathrm{r}}=1\right)$, 4- silicon $\left(\mu_{\mathrm{r}}=1\right), 5$-PES white $\left(\mu_{\mathrm{r}}=1\right), 6$-communication antenna $\left(\mu_{\mathrm{r}}=1, \rho=14.410^{-8} \Omega \cdot \mathrm{m}\right)$, and 7-fill-level sensor coil $\left(\mu_{\mathrm{r}}=1, \rho=1.710^{-8} \Omega \cdot \mathrm{m}\right)$. The antenna and fill-level sensor were modeled as stranded coils elements. In order to include the electronic circuit in the calculation, the FE model was fully coupled with an idealized capacitor element (shown on the right). The capacitance $\mathrm{C}_{\mathrm{p}}$ was chosen to tune the antenna at $13.56 \mathrm{MHz}$ (communication frequency) and the fill-level sensor at $100 \mathrm{kHz}$.

tuning of $13.56 \mathrm{MHz}$, which is the frequency used for the communication with the control unit (CU). The fill-level sensor capacitor was set for a tuning of $100 \mathrm{kHz}$. The niobium antenna with 2 windings and the copper fill-level sensor with 88 windings were modeled using stranded coil elements.

The boundary conditions used for solving the model were chosen to represent the MRI magnetic field. The field direction was aligned with the model's axis of symmetry (Fig. 1). This is the worst case situation for the pump's electronics since the field orientation is perpendicular to the antenna and fill-level sensor surfaces. The amplitude of the RF field used in this study was $30 \mu \mathrm{T}$, which corresponds to the maximal field produced by the body coil of a typical 1.5T MRI system (Philips Integra System). The magnitude of the gradient fields of an MRI device varies from zero at the isocenter to a maximum at the wall of the bore. To model the worst case situation, the fields were calculated for the pump placed in the most eccentric region of the MRI imaging space. The magnitudes of the gradient fields used for the simulations are given in Table I.

The commercial FE package ANSYS Emag 9 (ANSYS Inc., Canonsburg, PA) was used for the simulations. Harmonic analyses were performed to model the RF situation and transient analyses were used to solve the gradient field problem.

\section{FE Model Validation Step\#1-Laboratory}

An initial validation of the numerical results of the model was made by measuring the induced voltages in the pump's antenna by the RF emissions produced by the CU's loop antenna. This initial FE model validation compared the induced voltages measured experimentally at the pump unit (PU) antenna using an oscilloscope (Agilent 54642D Oscilloscope, Agilent Technologies, Palo Alto, CA) with the voltage obtained from the simulation. The experimental tuning of the system also accounted for the approximately $10 \mathrm{pF}$ parallel capacitance of the oscilloscope probe. With this setup, a direct comparison between experimental results and numerical calculations could be made. 
TABLE I

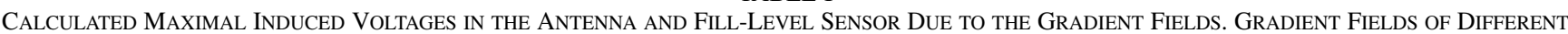
EXISTING MRI DEVICES AND CORRESPONDING GRAdIENT FIELDS USED FOR THE SiMUlations ARE PRESENTED. For THE CALCULATIONS, THE PUMP POSITION Was at $45 \mathrm{~cm}$ From the Bore Center. The Values Chosen for the Calculations Always Correspond to the Worst Case Situation

\begin{tabular}{|l|c|c|c|c||c|c|}
\hline & \multicolumn{3}{|c||}{ Gradient fields description } & \multicolumn{2}{c|}{ Induced Voltages (V) } \\
\cline { 2 - 7 } & $\begin{array}{c}\text { Max. field } \\
(\mathrm{mT} / \mathrm{m})\end{array}$ & $\begin{array}{c}\text { Slew Rate } \\
(\mathrm{T} / \mathrm{m} / \mathrm{s})\end{array}$ & $\mathrm{G}_{\mathrm{z}}(\mathrm{mT})$ & $\begin{array}{c}\text { Switch } \\
\text { time }(\mathrm{ms})\end{array}$ & Antenna & $\begin{array}{c}\text { Fill Level } \\
\text { Sensor }\end{array}$ \\
\hline 0.23T Philips Panorama & 21 & 50 & 9.5 & 0.4 & $0.2 \mathrm{~V}$ & $3.0 \mathrm{~V}$ \\
\hline 0.35T Siemens Magnetom & 24 & 55 & 10.8 & 0.4 & $0.2 \mathrm{~V}$ & $3.5 \mathrm{~V}$ \\
\hline 1.5T Philips Intera & 30 & 150 & 13.5 & 0.2 & $0.5 \mathrm{~V}$ & $7.6 \mathrm{~V}$ \\
\hline 3.0T Philips Achieva & 40 & 200 & 18 & 0.2 & $0.7 \mathrm{~V}$ & $11.3 \mathrm{~V}$ \\
\hline
\end{tabular}

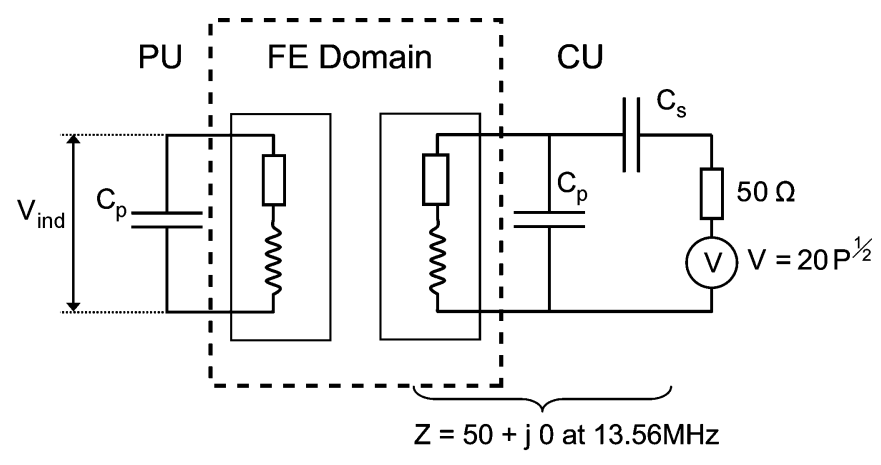

Fig. 2. Schematic representation of the circuit used to model the laboratory setup used for the FE model validation. The PU side is the same as for the simulation of the MRI situation. The CU side was designed to precisely model the experimental condition. An RF generator produced an oscillating voltage corresponding to different emission powers. The $50-\Omega$ internal resistance of the generator was also modeled. A matching circuit of two capacitors $\left(\mathrm{C}_{\mathrm{p}}\right.$ and $\left.\mathrm{C}_{\mathrm{s}}\right)$ was considered in order to ensure the resonance condition as well as a $50 \Omega$ matching of the complete circuit. The induced voltages $V_{\text {ind }}$ were calculated and measured experimentally with an oscilloscope.

To perform this validation a representation of the CU antenna was added to the FE model. The antenna was represented as a massive circular conductor with a radius of $65 \mathrm{~mm}$. The electronic circuit connected to the $\mathrm{CU}$ antenna which is used to generate the RF field was also represented in the FE model (Fig. 2). The CU's RF generator was modeled as producing an alternating voltage at $13.56 \mathrm{MHz}$ with a magnitude that corresponds to the different emission powers available $(0-3 \mathrm{~W})$. No modifications were made to the electronic circuit of the pump unit.

The experimental setup used for this validation consisted of an empty titanium pump case with its antenna. A signal generator was connected to the emitting loop antenna, which was placed around the pump, and used to produce RF fields at predefined power levels.

Two different tuning conditions were used in the validation to verify that the FE model captures the effect of de-tuning of the pump's communication system. In the first case, the capacitor's elements were set to model resonance frequencies of 13.56 MHz separately in the pump and CUs. This simulates a situation where these two devices are far away from each other. The second case simulates the situation of the antennas being brought into close proximity, where a de-tuning of both antennas is observed due to mutual induction. In this case, the capacitor elements were chosen to have both antennas tuned simultaneously to $13.56 \mathrm{MHz}$ while in their testing position. Additionally, the capacitor elements had to meet a $50 \Omega$ impedance matching
TABLE II

MEASURED INDUCED VOLTAGES IN THE ANTENNA FOR THE RF FIELDS PRODUCED By DiFFERENT MRI SCANNERS. MRI SEQUENCES WERE CHOSEN Without GRADIENT FieldS. These Results ARE COMPARED With FE CAlCulations CORRESPONDING to the SAME Situations. Note That the Results ARe Given at the Level of the $\mathrm{C}_{3}$ Capacitor (Fig. 3) and Not at the Antenna Level

\begin{tabular}{|l|c|c|}
\hline \multirow{2}{*}{} & \multicolumn{2}{|c|}{ RF field - B1 $=10 \mu \mathrm{T}$} \\
\cline { 2 - 3 } & MRI & FE \\
\hline 0.23T Philips Panorama & $0.3 \pm 0.1 \mathrm{~V}$ & $0.5 \mathrm{~V}$ \\
\hline 1.5T Philips Integra & $0.3 \pm 0.1 \mathrm{~V}$ & $0 \mathrm{~V}$ \\
\hline 3.0T Philips Achiva & $1.1 \pm 0.2 \mathrm{~V}$ & $0 \mathrm{~V}$ \\
\hline
\end{tabular}

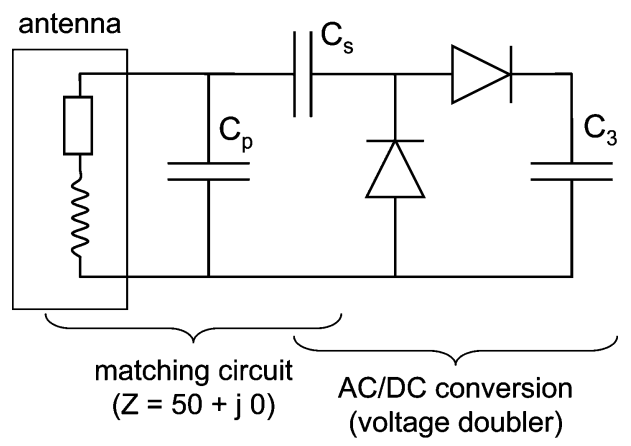

Fig. 3. Schematic representation of the circuit used for test measurements in MRI devices. First, a matching circuit was constructed in order to ensure the resonance condition. Then, an ac/dc conversion was made via two diodes elements (BAT 48) and the maximal induced voltages were stored in a $10-\mu \mathrm{F}$ capacitor element $C_{3}$. After the MRI imaging sequences, the accumulated voltage on $\mathrm{C}_{3}$ was measured with a simple digital multimeter.

condition at the $\mathrm{CU}$ antenna. Experimentally appropriate model parameters were selected using a Smith chart to plot the complex impedance of the circuit (Agilent 4369B Network Analyser, Agilent Technologies, Palo Alto, CA) while adjusting the values of different variable capacitor elements in the circuit.

\section{E. FE Model Validation Step\#2-MRI}

The second model validation experiment involved measuring the induced voltages in the antenna when the experimental apparatus was placed inside three representative clinical MRI scanners (Table II). A direct measurement of the voltages induced by the RF pulses of the MRI was not possible. Too much noise would have been generated by the external electrical connections. For this reason, a special electronic circuit was developed in order to capture the induced voltage without external electrical connections (Fig. 3). The circuit used a $10 \mu \mathrm{F}$ capacitor $\left(\mathrm{C}_{3}\right)$ to record the maximal voltage after ac to dc conversion. After exposing the test apparatus to the RF field of the MRI units, the voltage across the capacitor $\mathrm{C}_{3}$ was measured with 


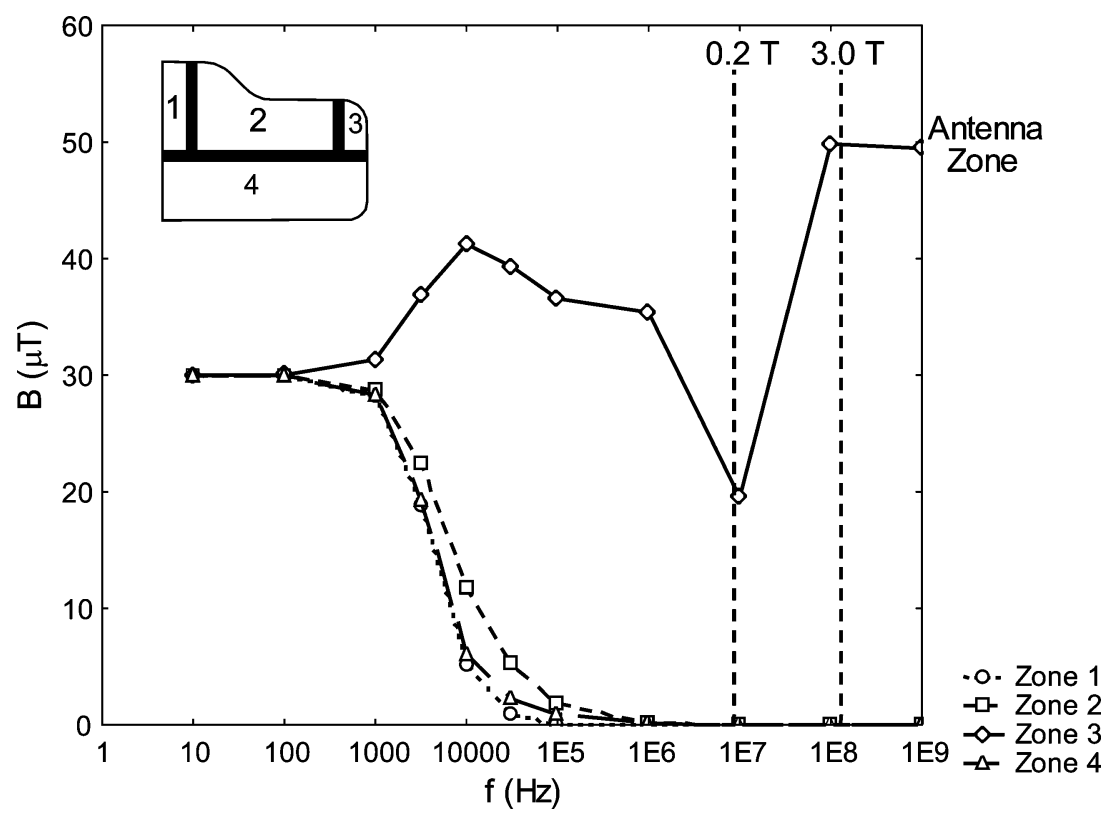

Fig. 4. Magnetic field at 4 points of the pump as a function of the field frequency. At the MRI working frequencies (between 0.2 and 3.0T), the field is null inside the titanium casing, but the field is increased in the region of the communication antenna.

a digital multimeter. The gradient magnetic fields of the MRI were disabled for this experiment.

A linear relationship between the amplitude of the induced voltages at the antenna and the direct voltages generated in the capacitor $\mathrm{C}_{3}$ was empirically determined as

$$
\mathrm{V}_{\text {antenna }}=0.67 \bullet \mathrm{V}_{\mathrm{C} 3}+1.1 \mathrm{~V} \text {. }
$$

The $\mathrm{R}^{2}$ value of the linear regression used to determine this relationship was 0.99 . This value expresses the proportion of data variance that can be explained by the regression; $A s R^{2}$ approaches unity, the regression approaches a perfect fit. The baseline signal of about $1 \mathrm{~V}$ is due to the forward voltage and reverse leakage of the diodes. The forward voltage of the diode used in this study (BAT 48) can be up to $0.9 \mathrm{~V}$.

\section{RESULTS}

\section{A. FE Simulations of MRI Induced Voltages}

The FE model was used to calculate the magnetic field at different locations inside the pump (Fig. 4). At the working frequencies of the MRI, the inside of the pump is perfectly magnetically shielded $(\mathrm{B}<0.02 \mu \mathrm{T})$. In contrast, the region surrounding the antenna, which is outside the titanium case, is subjected to magnetic fields that are higher than the main RF field $(\mathrm{B} \approx 70 \mu \mathrm{T})$. The magnetic shielding phenomenon that was observed inside the pump casing is a result of eddy currents that are generated on the surface of the case. These eddy currents concentrate at the sharp edges of the pump case, but with an amplitude of less than $1.5 \mathrm{~A} / \mathrm{mm}^{2}$ for a 3.0T MRI

The induced voltages in the antenna and fill-level sensor that were calculated for corresponding frequencies of the different
MRI devices are shown in Fig. 5. The estimated induced voltages for the fill-level sensor were insignificant and the induced voltages in the antenna were less than $1 \mathrm{~V}$ for both 1.5 and 3.0T machines. For the 0.3T MRI machine the induced voltages were up to $20 \mathrm{~V}$. The highest induced voltage calculated was about $70 \mathrm{~V}$, but no existing MRI scanner uses a $0.318 \mathrm{~T}$ magnet that would produce such a voltage.

The induced voltages were also calculated for pump devices subjected to gradient fields (Table I). In this situation, the induced voltages in the pump's antenna were negligible $(<1 \mathrm{~V})$, but those in the fill-level sensor were significant-up to $11 \mathrm{~V}$. The shielding effect of the titanium casing is less effective against gradient fields and the electronics inside the pump are therefore less protected against the affect of these fields.

\section{B. FE Model Validation Step\#1-Laboratory}

The comparison of experimentally measured induced voltages at the pump's antenna with corresponding numerical estimations is presented in Fig. 6 for the two different tuning methods. The numerical model gave estimates of the experimental data with a maximal error of less than $3 \mathrm{~V}$. The induced voltages obtained in the separate tuning situation were about two times lower than those for the combined situation. This voltage decrease illustrates the de-tuning caused by the mutual induction phenomenon.

\section{FE Model Validation Step\#2-MRI}

The experimental measurements of induced voltages from different MRI devices were compared to the numerical estimates for the corresponding situations (Table II). The values presented were for voltages at the $\mathrm{C}_{3}$ component and not at the location of the antenna. This observation explains the values of $0 \mathrm{~V}$ obtained with the numerical model at 1.5 and 3.0T because 


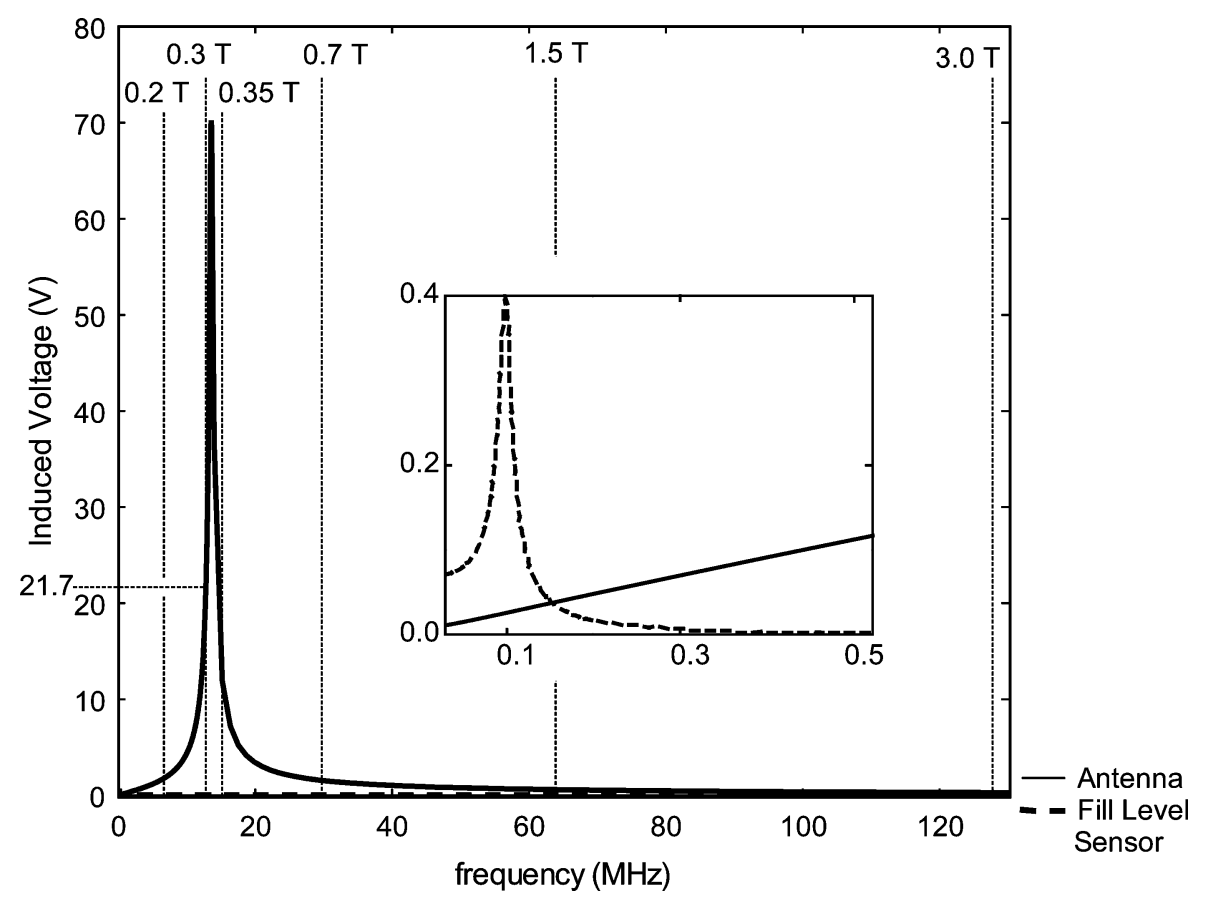

Fig. 5. Induced voltages in the antenna and fill-level sensor as a function of the RF field frequency. The induced voltages in the fill-level sensor are shown in a detailed view around $100 \mathrm{kHz}$. Induced voltages are very low at 1.5 and $3.0 \mathrm{~T}(<1 \mathrm{~V})$, but is higher for $0.3 \mathrm{~T}$ MRI (about $20 \mathrm{~V})$.
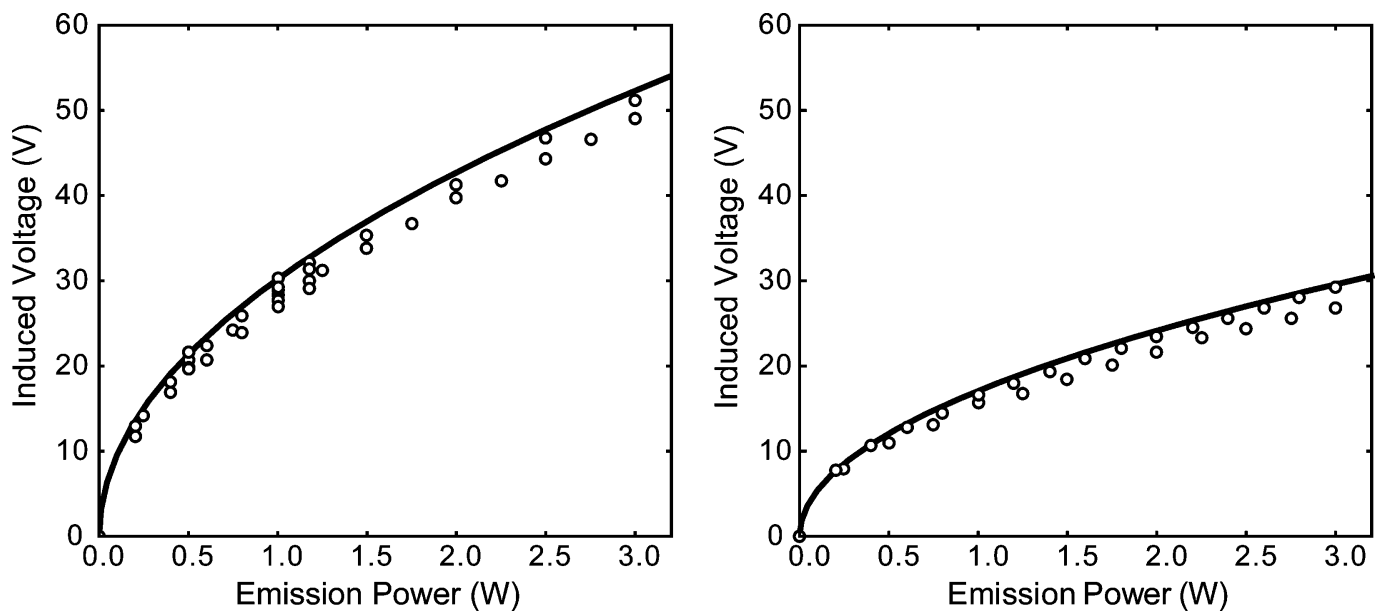

Fig. 6. Results of the laboratory measurements. The induced voltages for different CUs emission powers were measured experimentally (circles) and calculated (lines). The graph on the left represents the combined tuning situation and the graph on the right represents the separate tuning situation. Experimental and numerical results are in agreement with an error of about $3 \mathrm{~V}$ or $5 \%$.

the calculated values at the antenna were below the diode's forward voltage threshold as shown in (2).

\section{DISCUSSION}

A novel methodology was developed for performing a preliminary evaluation of the MRI-safety of active implants. This methodology was used to locate any potential sources of incompatibility between an active implantable pump and MRI. An FE numerical model was developed to simulate the influence of the time-varying magnetic fields of an MRI machine and estimate the induced voltage in the critical electric components of the pump. The results of this study indicated that there is a potential operational safety problem when a $0.3 \mathrm{~T}$ MRI is used. This problem is due to the effects of the RF pulses on the pump antenna. Another potential problem was noted when a 3.0T MRI was used and this is due to the interaction between the gradient fields and the fill-level sensor. This study aided the understanding of these possible incompatibilities and enabled corrective measures to be taken.

The FE simulations showed that the titanium casing enclosing the pump provided a very good shielding to the RF electromagnetic field. This shielding offers protection for all the electronic components inside the pump. This observation is confirmed by the induced voltages calculated for the fill-level sensor. This coil has almost the same size as the antenna, but has 40 times more 
windings and is located inside the pump. For this coil, only negligible induced voltages were calculated during RF analyses.

The shielding is less effective against gradient fields. The reduced protection is the result of the transient nature of the gradient fields and the fact that their frequency of about $5 \mathrm{kHz}$ (at $1.5 \mathrm{~T}$ ) is much smaller than the frequency of the RF-field. As a consequence, the electronic components inside the pump are exposed to the gradient fields, explaining the relatively high induced voltages calculated for the fill-level sensor.

Induced voltages are associated with any time varying fields and these can arise from patient motion through a nonhomogeneous magnetic field. The principal magnetic field produced by the MRI is designed to be perfectly homogeneous inside the device, but it is no longer homogeneous outside the MRI bore. The field decrease from its maximal value within the bore to zero a few meters away from the magnet. For a $1.5 \mathrm{~T}$ device, a maximal spatial variation of approximately $30 \mathrm{mT} / \mathrm{cm}$ takes place about $80 \mathrm{~cm}$ away from the magnet. Thus, if a patient lying on the table is moved at $1.5 \mathrm{~m} / \mathrm{s}$ (walking speed) through this field, he is submitted to a maximal time varied magnetic field that is six times lower than gradient fields of a 0.23T MRI. For this situation, induced voltages of less than $1 \mathrm{~V}$ were calculated in the fill-level sensor. These very small voltages do not represent any risk for the electronic safety of the device.

According to the FE estimations, the worst case for the electronic components occurs in low field-strength scanners. Induced voltages up to $20 \mathrm{~V}$ were calculated when a $0.3 \mathrm{~T}$ primary MRI field was used. This result might seem counterintuitive, but the RF frequency of a scanner with this field strength is close to the communication frequency of the antenna. In theory, identical gradient fields can be used for all static field strengths, but as seen in Table I, the gradient field strengths used for higher static fields also tend to be higher and these higher fields result in higher induced voltages. As a consequence, the safety of the device should be tested in a full range of MRI devices; high field MRIs to evaluate the risks for the electronic components resulting from gradient fields, and low field MRIs to evaluate the risk due to RF fields.

This study demonstrated a good agreement between laboratory measurements and numerical estimations. It is important to note that the model was able to simulate both the combined and the separate tuning situations. This result is a strong validation of the general model behavior and of the FE/idealized circuit element coupling. The differences between the numerical and the experimental results were less than $3 \mathrm{~V}$. The error level is sufficient low to ensure qualitatively meaningful results and quantitatively accurate estimation of induced voltages.

The MRI validation of the FE simulations also demonstrates the agreement between the model and the experimental measurements. However, the model validation has only a few comparison points because it is difficult to find MRI devices encompassing all possible different field strengths. Moreover, this validation is less accurate than the laboratory evaluation, because for the MRI measurements, the induced voltages were measured in an indirect way. Additional errors may have been introduced during the ac/dc conversion of the induced voltages. Moreover, induced voltages below the diode forward voltage threshold were not measurable [see (2)].
To our knowledge, this paper is the first to describe a method able to evaluate the MRI-safety of sensitive electronic components of a device before its effective production. Previously performed numerical studies have only focused on the evaluation of the amount of RF energy absorbed by the tissues around different implants (SAR), but not on the device electronics. Schueler et al. [2] calculated the magnetic field around an aluminum spheroid implant as well as the eddy currents on this implant surface. Field distribution around this spheroid implant was similar to the results obtained in the present work, but the RF field magnitude was not specified. Thus quantitative comparisons are not possible. Our results suggest that the electronics inside the pump will be protected from the MRI induced voltages. Our results confirm the observations that have been made with some modern implanted active devices, which have not failed during scanning [6], [9], [18].

There are some limitations to this FE model. A low-frequency approximation was used for the calculations. This approximation is perfectly valid for the simulation performed with the pump in air presented in this paper. However, this estimation is no longer valid for the RF calculation of the pump in a physiological solution or surrounded by biological tissues. In these cases, the high dielectric constant of the medium leads to a decrease in the electromagnetic wavelength to approximately five times the pump's dimension. On the other hand, these calculations were not focused on the effect of the electromagnetic fields on the tissues surrounding the pump, but rather to evaluate the risks for the electronic components. From this point of view, the results should not change significantly with the pump in physiological fluid as the transmission frequency remains the same as in air, and the amplitude of the RF field is slightly smaller due to the shielding effect of the water. Thus, the induced voltages generated by the RF fields would be expected to be slightly smaller with the pump implanted into biological tissues.

The MRI magnetic fields used for the simulations are idealized approximations of the real fields present in MRI devices. The RF field was represented as a vector oscillating in the vertical direction. In a real MRI, this field rotates around the principal bore axis with a rotation frequency equal to the gyromagnetic ratio times the field strength. However, our approximation is valid for the calculation of the induced voltages in the antenna, because only the product of the magnetic vector with the antenna/coil surface normal vector is important. This product is the same for both rotating field and the constant orientation field. Moreover, the maximum possible emission power was used for the RF field simulation. Consequently, the calculated values represent the worst case scenario and, correspondingly, the upper limit of the induced voltages.

The gradient fields used in the calculations were also idealized. A precise definition of the gradient field strength and orientation is difficult to obtain for existing scanners. In addition, the concomitant fields to the desired gradient in the $\mathrm{z}$ direction increase the complication of the problem [19]. For this reason, the worst case situation was modeled. Gradient fields used for the calculations were obtained with both slew rate and maximum fields at their extreme values, which is an impossible condition in practical situations. Moreover, the pump was positioned in 
the most eccentric region of the MRI sample space, which is unlikely to be achieved in practice.

Obviously, the electronic circuit connected to the antenna only represents the resonant part of a more complex communication circuit. However, this allows for a good evaluation of the worst case situation. The rest of the circuit adds resistive elements to this resonant part, which decrease the induced voltages in the circuit. The situation modeled here corresponds to an upper limit of the induced voltages at the level of the antenna. This information may then be used as a superior limit to evaluate the voltage at different points of the complete electronic circuit.

This study showed that a numerical model can be used to estimate the electronic MRI-safety issues for active implants. We focused on the interactions between the electronic components of the implant and the MRI magnetic fields, but the FE method may also be used to evaluate risks associated with other physical phenomena, not only those associated with active implants. These risks may include the mechanical forces acting upon the implant, tissue heating due to eddy currents flowing on the metallic surface and the electromagnetic energy deposited in the tissues around the implant.

The method presented in this paper can be used to help achieve a robust MRI-safe design of the critical electric components of active implants. Its results can indicate the need for protective circuits, required to ensure that any induced voltages have minimal effect. Numerical analysis of this type could be used to evaluate the potential safety issues early in the development cycle, when critical design decisions and modifications are less costly in time and material.

In this study, no operational safety problem was found for the investigated pump during 1.5 and 3.0T MRI scanning. The high induced voltages calculated during $0.3 \mathrm{~T}$ scanning could be filtered with an appropriate protection circuit. Obviously, real experimental testing will always be required before finally declaring an implant as MRI-safe, but the combination of numerical simulations and experimental testing can be a powerful tool in the development of MRI-safe implants.

\section{REFERENCES}

[1] O. G. Anfinsen, R. F. Berntsen, H. Aass, E. Kongsgaard, and J. P. Amlie, "Implantable cardioverter defibrillator dysfunction during and after magnetic resonance imaging," Pacing Clin. Electrophysiol., vol. 25, no. 9, pp. 1400-1402, 2002.

[2] B. A. Schueler, T. B. Parrish, J. C. Lin, B. E. Hammer, B. J. Pangrle, E. R. Ritenour, J. Kucharczyk, and C. L. Truwit, "MRI compatibility and visibility assessment of implantable medical devices," J. Magn. Reson. Imag., vol. 9, no. 4, pp. 596-603, 1999.

[3] FDA Medical Device Report (MDR) Records \#351516, \#748838, $\# 175218$, and \#125938.

[4] W. Irnich, B. Irnich, C. Bartsch, W. A. Stertmann, H. Gufler, and G. Weiler, "Do we need pacemakers resistant to magnetic resonance imaging?," Europace., vol. 7, no. 4, pp. 353-365, 2005.

[5] R. Luechinger, F. Duru, M. B. Scheidegger, P. Boesiger, and R. Candinas, "Force and torque effects of a 1.5-Tesla MRI scanner on cardiac pacemakers and ICDs," Pacing Clin. Electrophysiol., vol. 24, no. 2, pp. 199-205, 2001.

[6] E. T. Martin, J. A. Coman, F. G. Shellock, C. C. Pulling, R. Fair, and K. Jenkins, "Magnetic resonance imaging and cardiac pacemaker safety at 1.5-Tesla," J. Am. Coll. Cardiol., vol. 43, no. 7, pp. 1315-1324, 2004.

[7] F. G. Shellock and S. M. Morisoli, "Ex vivo evaluation of ferromagnetism, heating, and artifacts produced by heart valve prostheses exposed to a 1.5-T MR system," J. Magn. Reson. Imag., vol. 4, no. 5, pp. 756-758, 1994.
[8] R. Luechinger, V. A. Zeijlemaker, E. M. Pedersen, P. Mortensen, E. Falk, F. Duru, R. Candinas, and P. Boesiger, "In vivo heating of pacemaker leads during magnetic resonance imaging," Eur. Heart J., vol. 26, no. 4, pp. 376-383, 2005.

[9] T. Sommer, C. Vahlhaus, G. Lauck, A. von Smekal, M. Reinke, U. Hofer, W. Block, F. Traber, C. Schneider, J. Gieseke, W. Jung, and H. Schild, "MR imaging and cardiac pacemakers: in-vitro evaluation and in-vivo studies in 51 patients at 0.5 T," Radiology, vol. 215, no. 3, pp. 869-879, 2000.

[10] A. R. Rezai, D. Finelli, J. A. Nyenhuis, G. Hrdlicka, J. Tkach, A. Sharan, P. Rugieri, P. H. Stypulkowski, and F. G. Shellock, "Neurostimulation systems for deep brain stimulation: in vitro evaluation of magnetic resonance imaging-related heating at 1.5 tesla," J. Magn. Reson. Imag., vol. 15, no. 3, pp. 241-250, 2002.

[11] V. M. Tronnier, A. Staubert, S. Hahnel, and A. Sarem-Aslani, "Magnetic resonance imaging with implanted neurostimulators: an in vitro and in vivo study," Neurosurgery, vol. 44, no. 1, pp. 118-125, 1999.

[12] A. L. Kovac, B. Swanson, C. Elliott, and L. Wetzel, "Effect of distance and infusion rate on operation of Medfusion 2010 infusion pump during magnetic resonance imaging," J. Clin. Anesth., vol. 14, no. 4, pp. 246-251, 2002.

[13] R. Von Roemeling, R. M. Lanning, and F. A. Eames, "MR imaging of patients with implanted drug infusion pumps," J. Magn. Reson. Imag., vol. 1, no. 1, pp. 77-81, 1991.

[14] L. M. Angelone, A. Potthast, F. Segonne, S. Iwaki, J. W. Belliveau, and G. Bonmassar, "Metallic electrodes and leads in simultaneous EEGMRI: specific absorption rate (SAR) simulation studies," Bioelectromagnetics, vol. 25, no. 4, pp. 285-295, 2004.

[15] C. M. Collins, W. Liu, J. Wang, R. Gruetter, J. T. Vaughan, K. Ugurbil, and M. B. Smith, "Temperature and SAR calculations for a human head within volume and surface coils at 64 and $300 \mathrm{MHz}$," J. Magn. Reson. Imag., vol. 19, no. 5, pp. 650-656, 2004.

[16] H. S. Ho, "Safety of metallic implants in magnetic resonance imaging," J.Magn Reson.Imaging, vol. 14, no. 4, pp. 472-477, 2001.

[17] D. Simunic, P. Wach, W. Renhart, and R. Stollberger, "Spatial distribution of high-frequency electromagnetic energy in human head during MRI: numerical results and measurements," IEEE Trans. Biomed. Eng., vol. 43, no. 1, pp. 88-94, Jan. 1996.

[18] F. Duru, R. Luechinger, M. B. Scheidegger, T. F. Luscher, P. Boesiger, and R. Candinas, "Pacing in magnetic resonance imaging environment: clinical and technical considerations on compatibility," Eur. Heart J., vol. 22, no. 2, pp. 113-124, 2001.

[19] D. G. Norris and J. M. Hutchison, "Concomitant magnetic field gradients and their effects on imaging at low magnetic field strengths," Magn. Reson. Imag., vol. 8, no. 1, pp. 33-37, 1990.

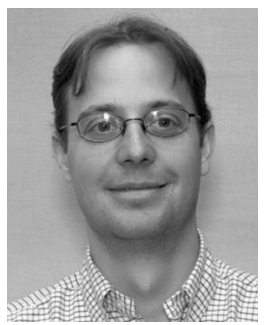

Philippe Büchler received the M.S. degree in physics and the Ph.D. degree from the Swiss Federal Institute of Technology, Lausanne, Switzerland, in 1998 and 2002, respectively.

After working as a Research Scientist at the Swiss Federal Institute of Technology, he joined the MEM Research Center, University of Bern, Bern, Switzerland and became head of the Computational Bioengineering group in 2006. His research interests include the application of numerical methods to study skeletal biomechanics as well as avascular soft tissues. Recently, electromagnetic field simulation has expanded his fields of research.

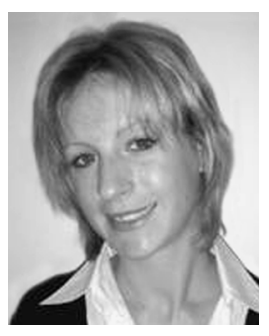

Anne Simon received the B.S. degree in micro-engineering with specialization in project management, sensor engineering, and optics from the University of Applied Sciences of Bern, Bern, Switzerland, in 2004.

After two years experience on the assessment of MRI-compatibility of active implants at the University of Applied Sciences in collaboration with Codman (a Johnson \& Johnson company), Le Locle, Switzerland, she is currently with the Quality Department of Stryker Osteosynthesis, Selzach, Switzerland. Her interests include quality management of orthopedic implants. 


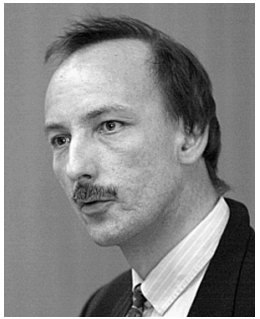

Jürgen Burger received the diploma and doctoral degrees in physics from the University Erlangen-Nürnberg, Erlangen, Germany, in 1987 and 1993.

He is currently Professor for Microtechnology and Biomedical Engineering at the Bern University of Applied Sciences, Bern, Switzerland, and Co-Program Director of the biomedical engineering master program of the University of Bern. His research interests include intelligent surgical instruments and implantable devices in neurosurgery, ophthalmology, otology, osteosynthesis, and orthopaedics.

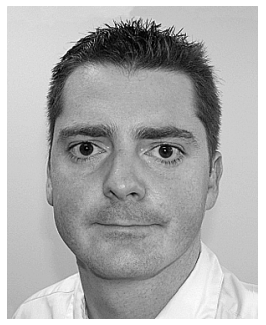

Alec Ginggen was born in Lausanne, Switzerland, in 1972. He received the M.S. degree in physics from the Swiss Federal Institute of Technology in Lausanne, Switzerland, in 1997.

Since then he has been with Codman (a Johnson \& Johnson company), Le Locle, Switzerland. He is currently heading the Microsystems Research and Development group in Le Locle, Switzerland. His research interests include biomedical applications of microsystems and telemetry.

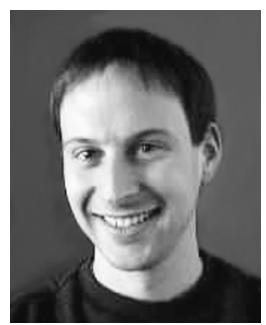

Rocco Crivelli was born in Locarno, Switzerland, in 1976. He received the M.S. degree in micro-technology from the Swiss Federal Institute of Technology, Lausanne, Switzerland, in 2000.

In 2000, he joined the $R \& D$ department of Codman (a Johnson \& Johnson company), Le Locle, Switzerland. Since then he has worked on the realization of passive wireless telemetry links for the communication between implanted medical devices and an external control unit. He is currently a Staff Engineer and continues to drive the development of hardware and software for active implants.

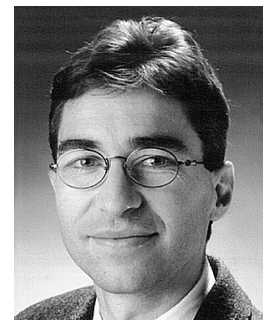

Yanik Tardy was born in Lausanne, Switzerland, in 1962. In 1992, he received the Ph.D. degree in physics from the Swiss Federal Institute of Technology, Lausanne.

He spent two years as a Postdoctoral Fellow with the Department of Mechanical Engineering at the Massachusetts Institute of Technology (MIT), Cambridge, working on cellular mechanics. In 1994, he created a vascular cell mechanics research group with the Laboratory of Biomedical Engineering at the Swiss Federal Institute of Technology. In 1998 he was appointed as Research \& Development Manager with Codman, (a Johnson \& Johnson company), Le Locle, Switzerland, to lead design activities for medical implants out of a manufacturing site based in Neuchâtel, Switzerland. He is currently Director of Operations and $R \& D$ for a business unit in charge of developing and manufacturing implantable pumps.

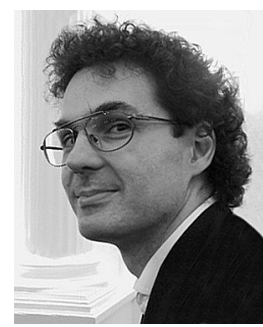

Roger Luechinger received the Ph.D. degree in magnetic resonance imaging from the institute for Biomedical Engineering of the University and ETH Zurich, Zürich, Switzerland, in 2002. The topics of his thesis were safety aspects of pacemaker in MRI.

In 2003 he became coordinator MRI Center of the strategic excellence projects of the ETH Zurich. His interests lie beside the safety aspects of MRI in the area of high resolution MR imaging of bones and cartilage of the lower and upper extremities.

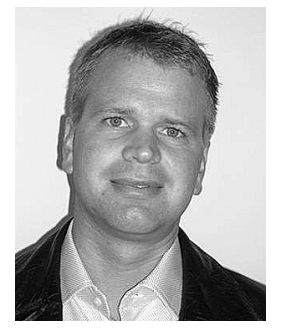

Sigbjørn Olsen received the B.S. and M.S. degrees in mechanical engineering from University of Trondheim, Trondheim, Norway, and Royal Melbourne Institute of Technology, Melbourne, Australia, respectively, before moving into biomechanics. $\mathrm{He}$ completed the thesis on numerical techniques for analysis in orthopedics for the Ph.D. degree in biomechanics at Queensland University of Technology, Brisbane, Australia.

Upon joining the MEM Research Center in Switzerland, he was given the opportunity to expand the range of applications for these techniques to include a range of research topics in medical technology. 